\title{
Improving women's experience during speculum examinations at routine gynaecological visits: randomised clinical trial
}

Dean A Seehusen, Dawn R Johnson, J Scott Earwood, Sankar N Sethuraman, Jamie Cornali, Kelly Gillespie, Maria

Doria, Edwin Farnell IV, Jason Lanham

\begin{abstract}
Objectives To determine if a standardised method of leg positioning without stirrups reduces the physical discomfort and sense of vulnerability and increases the sense of control among women undergoing speculum examination as part of a routine gynaecological examination.

Design Randomised clinical trial.

Setting Family medicine outpatient clinic.

Patients 197 adult women undergoing routine gynaecological examination and cervical smear.

Intervention Examination with or without stirrups.

Main outcome measures Women's perceived levels of physical discomfort, sense of vulnerability, and sense of control during the examination, measured on $100 \mathrm{~mm}$ visual analogue scales. Results Women undergoing examination without stirrups had a reduction in mean sense of vulnerability from 23.6 to 13.1 (95\% confidence interval of the difference -16.6 to -4.4 ). Mean physical discomfort was reduced from 30.4 to 17.2 $(-19.7$ to -6.8$)$. There was no significant reduction in sense of loss of control.

Conclusion Women should be able to have gynaecological examinations without using stirrups to reduce the stress associated with speculum examinations.

Trial registration US Army Central Investigation Regulatory Office. Trial No DDEAMC 05-11.

\section{Introduction}

Compliance rates with cervical screening vary, and embarrassment and fear of pain during examination have been reported as potential barriers to screening. ${ }^{1-3}$ Reducing these barriers may improve screening rates. ${ }^{3}$ Whereas in countries such as the United Kingdom, Australia, and New Zealand speculum examinations are done without stirrups with the woman in the dorsal or lateral positions, in the United States medical providers are trained to do gynaecological examinations with women in the dorsal lithotomy position in stirrups. We examined the use of an alternative positioning to improve women's experience with speculum examinations at routine gynaecological visits.
\end{abstract}

\section{Methods}

Assignment, masking, and flow-Women aged $\geq 18$ years presenting for a routine gynaecological examination in a family medicine clinic were eligible for inclusion. We excluded women who were pregnant, had vaginal complaints, or were unable to consent for themselves. Participants were enrolled from January to August 2005. Women were randomised into "stirrup" and "no stirrup" groups by assigning study numbers to one of the two groups using an online random number generator and enclosing the assignments in envelopes. The envelopes were opened, revealing the method to be used, after the woman agreed to participate (fig 1). This study was powered to have an $80 \%$ chance of detecting a $5 \mathrm{~mm}$ difference in sense of vulnerability or discomfort, with an SD of $22 \mathrm{~mm}$ (determined by pilot study), on a $100 \mathrm{~mm}$ visual analogue scale. We originally intended to include 300 women but we stopped after a planned interim analysis of 200 patients.

Protocol-Patients in the control group were examined with their heels placed in uncovered metal stirrups at $30-45^{\circ}$ angles to the examination table. The intervention group were examined with their feet placed on the corners of a fully deployed table extension. All women were draped with a full sized sheet in a standardised manner that maximised coverage of the woman and allowed visualisation of the perineum (fig 2). Medium Graves' plastic speculums and an external light source were used for all examinations.

Provider training-We recruited eight providers from within the clinic: five staff physicians, two resident physicians, and one family nurse practitioner (five men and three women). All providers were taught how to position and cover the women with a combination of individual teaching, still photographs, and a short video.

Outcomes measured-We collected demographic data for each participant. Race, as identified by the women from a list provided, was recorded because it has often been looked at in

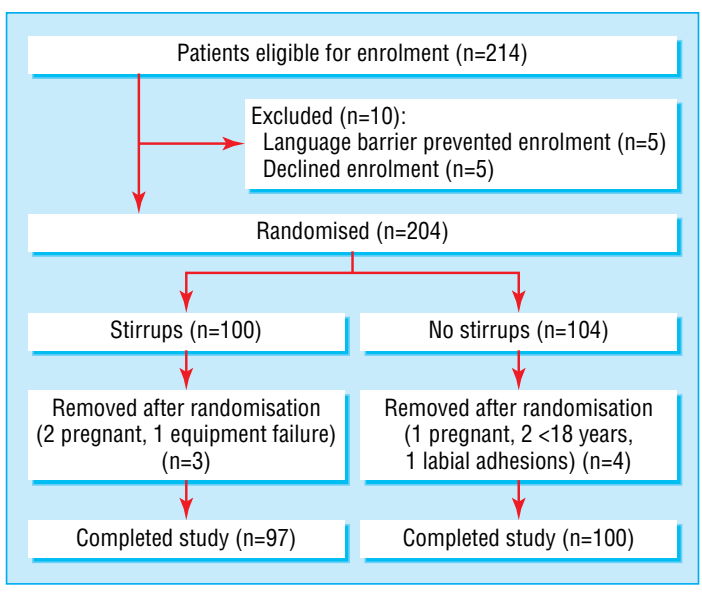

Fig 1 Patient flow through the study 


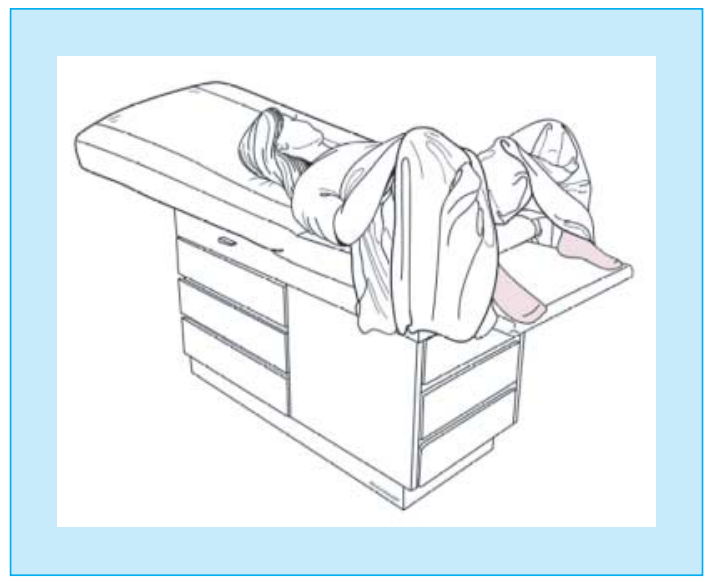

Fig 2 Positioning of women with draping for examination without stirrups (drawn by Jordan Mastrodonato)

relation to satisfaction or compliance with cervical smears. Afterwards, women completed a questionnaire regarding the examination. We used $100 \mathrm{~mm}$ visual analogue scales to measure physical discomfort, sense of vulnerability, and sense of loss of control. Study identification numbers were used to preserve anonymity of the respondents. We had previously piloted the questionnaire on 25 women. We assessed the quality of smears according to adequacy and the presence or absence of endocervical cells reported in the pathology reports.

Data handling-Two researchers (DAS and DRJ) entered the demographic data and results of the smear tests on a spreadsheet. An independent observer measured and entered the visual analogue scores on a separate spreadsheet in a blinded manner.

Analysis-We used SPSS version 11.0 for analysis. In the preliminary analyses we used $\chi^{2}$ tests for categorical data and $t$ tests for independent samples for continuous data. We tested the hypothesis with the $t$ test for independent samples to determine differences in outcome variables.

\section{Results}

We recruited and randomised 204 women. After randomisation, seven women were removed from the study: five did not meet enrolment criteria (three were pregnant and two were adolescents), one woman had labial adhesions that prevented insertion of the speculum, and there was one equipment failure caused by a faulty stirrup. Table 1 shows the baseline characteristics of the remaining women (table 1).

Physical discomfort and sense of vulnerability were both significantly lower in the women examined without stirrups (table 2). Mean sense of vulnerability was $44 \%$ lower and mean physical discomfort was $43 \%$ lower. Sense of loss of control was not significantly different.

There were no significant differences in the quality of smears between the two groups. Only two smears were inadequate for evaluation (both from the no stirrups group, $\mathrm{P}=0.16$ ). Sixteen of the smears from the women examined without stirrups and 15 from the women examined with stirrups did not contain endocervical cells $(\mathrm{P}=0.84)$.

\section{Discussion}

Women undergoing gynaecological examination without stirrups report less physical discomfort and a reduced sense of vulnerability than women in stirrups.
Table 1 Baseline characteristics of women undergoing gynaecological examination with or without use of stirrups. Figures are numbers of women unless stated otherwise

\begin{tabular}{|c|c|c|}
\hline Characteristic & No stirrups $(n=100)$ & Stirrups $(\mathrm{n}=97)$ \\
\hline Mean (SD) age (years) & $37.1(12.9)$ & 36.8 (12.3) \\
\hline Mean (SD) BMI & $28.3(5.7)$ & $27.7(5.3)$ \\
\hline \multicolumn{3}{|l|}{ Race: } \\
\hline White & 51 & 50 \\
\hline Black & 42 & 35 \\
\hline Hispanic & 2 & 4 \\
\hline Other & 2 & 4 \\
\hline Not reported & 3 & 4 \\
\hline \multicolumn{3}{|c|}{ No of previous cervical smears: } \\
\hline 0 & 1 & 1 \\
\hline $1-5$ & 19 & 20 \\
\hline $6-10$ & 16 & 10 \\
\hline$\geq 11$ & 61 & 62 \\
\hline Not reported & 3 & 4 \\
\hline Previous vaginal delivery & 68 & 64 \\
\hline \multicolumn{3}{|c|}{ Examiner who carried out smears: } \\
\hline 1 & 34 & 36 \\
\hline 2 & 16 & 21 \\
\hline 3 & 6 & 3 \\
\hline 4 & 25 & 17 \\
\hline 5 & 4 & 3 \\
\hline 6 & 8 & 11 \\
\hline 7 & 4 & 5 \\
\hline 8 & 3 & 1 \\
\hline Female examiner & 75 & 74 \\
\hline
\end{tabular}

BMI=body mass index.

\section{Weaknesses of this study}

Our outcome variables were broad categories. When answering about physical discomfort, were women measuring pain from speculum insertion, leg position, or both? These outcomes will need to be more precisely defined in future research. Only eight providers from the same practice were involved in this study.

\section{Meaning of this study}

Given that over 50 million cervical smears are done each year in the US, ${ }^{4}$ any reduction in the associated physical and psychological stress should prompt a change in clinical practice provided there are no associated negative implications. Previous studies have provided anecdotal evidence that women prefer examinations done without stirrups. ${ }^{5-7}$

The technique without stirrups used in this study is easy to learn, simple to perform, and does not affect the adequacy of examinations. Though Curtis reported that his rate of adequate smears went up when he switched to examination with stirrups, ${ }^{8}$ our results suggest that examination without stirrups does not affect adequacy, though a larger study would be needed to confirm this.

A sense of vulnerability or defencelessness is one of the reasons why women dislike pelvic examinations. ${ }^{2}$ Once a woman's feet are placed in stirrups, getting out is neither quick nor easy. Stirrups suspend the feet in the air and greatly reduce the ability to manoeuvre, which may give women a sense of being unable to protect themselves from potential danger. Women undergoing a

Table 2 Main outcome measures (mean (SD) score on $100 \mathrm{~mm}$ visual analogue scale) in 197 women examined with or without stirrups

\begin{tabular}{|c|c|c|c|}
\hline Outcome & No stirrups & Stirrups & Difference $(95 \% \mathrm{Cl})$ \\
\hline Sense of vulnerability & $13.1(16.3)$ & $23.6(25.8)$ & $-10.5(-16.6$ to -4.4$)$ \\
\hline Physical discomfort & $17.2(17.8)$ & $30.4(26.8)$ & $-13.2(-19.7$ to -6.8$)$ \\
\hline Sense of loss of control & $17.7(23.8)$ & $23.1(27.1)$ & $-5.4(-12.6$ to 1.8$)$ \\
\hline
\end{tabular}


cervical smear without stirrups are much freer to adjust the position of their feet and hips during the examination. The method we used in this study allows considerable internal or external rotation of the hips and the knees as well as supination of the feet. Each woman can manoeuvre her legs and feet into the position that is most comfortable and relaxing for her.

The level of physical discomfort reported by the stirrup group was similar to that in a previous study done on women presenting with abdominal pain or vaginal bleeding. ${ }^{9}$ In that study, patients had an average pain score of $33.8 \mathrm{~mm}$ on similar a visual analogue scale when they were examined by a woman and $38.8 \mathrm{~mm}$ when examined by a man.

Various interventions to reduce the physical and psychological distress associated with speculum examinations have been studied. Alternative positions, ${ }^{10}$ alternative speculums, ${ }^{11}$ alternative foot rests, ${ }^{12}$ self insertion of the speculum, ${ }^{13}$ and different types of gowns ${ }^{14}$ have shown varying degrees of improvement in pain, embarrassment, or anxiety. Reduction of physical discomfort and psychological distress are part of the motivation for the recent attempts to validate self collection methods for cervical cancer screening to eliminate the need for smears. ${ }^{15} 16$ Some studies have shown that beliefs about pain during the procedure can influence screening behaviours for some women. ${ }^{13}$

Respect for patients' preferences and ensuring physical comfort are core dimensions of patient centred care, one of the six domains of quality advocated by the Institute of Medicine.${ }^{17}$ Our findings suggest that examination without stirrups represents a more patient centred way to perform speculum examinations.

Contributors: DAS was responsible for conception and design of the study, collected and interpreted data, drafted the manuscript, and is guarantor. DRJ was responsible for conception and design of the study, collected data, and edited the manuscript. JSE collected data and drafted the manuscript. SNS carried out the statistical analysis, interpreted data, and critically edited

\section{What is already known on this topic}

Speculum examinations to collect cervical smears are the most commonly performed procedures on women

Women dislike undergoing speculum examinations because of fear of pain, embarrassment, or anxiety about feeling vulnerable during the examination

\section{What this study adds}

Women feel less vulnerable and experience less discomfort when examination is carried out without stirrups the manuscript. JC, KG, MD, EF, and JL collected data and edited the manuscript.

Funding: Uniformed Services Academy of Family Physicians.

Competing interests: None declared.

Ethical approval: This study underwent review for ethical considerations by the Institutional Review Board of Eisenhower Army Medical Center.

1 Kahn JA, Goodman E, Huang B, Slap GB, Emans SJ. Predictors of Papanicolaou smear return in a hospital-based adolescent and young adult clinic. Obstet Gynecol 2003:101:490-9.

2 Larsen M, Oldeide CC, Malterud K. Not so bad after all ... women's experiences of pelvic examinations. Fam Pract 1997; 14:148-52.

3 Hoyo C, Yarnall KSH, Skinner CS, Moorman PG, Sellers D, Reid L. Pain predicts nonadherence to Pap smear screening among middle-aged African American women. Prev Med 2005;41:439-45

4 National Cancer Institute. The Pap test: questions and answers. www.cancer.gov/ cancertopics/factsheet/Detection/Pap-test (accessed 20 Oct 2005).

5 Greiver M. No stirrups? Can Fam Physician 2001;47:1979.

6 Klassen D. Preference for the no-stirrup method. Can Fam Physician 2002;48:457.

7 Doig A. De facto evidence for the no-stirrup method. Can Fam Physician 2002;48:876.

8 Curtis P. Personal perspectives on collecting Papanicolaou smears or how I learned to use the stirrups.J Fam Pract 1991:33:345-7.

9 Moettus A, Sklar D, Tandberg D. The effect of physician gender on women's percieved pain and embarrassment during pelvic examination. Am J Emerg Med 1999;17:635-7.

10 Seymore C, DuRant RH, Jay S, Freeman D, Gomez L, Sharp C, et al. Influence of position during examination, and sex of examiner on patient anxiety during pelvic examination. J Pediatr 1986;108:312-7.

11 Thomas A, Weisberg E, Lieberman D, Fraser IS. A randomised contolled trial comparing a dilating vaginal speculum with a conventional bivalve speculum. Aust NZ J Obstet Gynaecol 2001;41:379-86.

12 Olson BK. Patient comfort during pelvic examination. New foot supports vs metal stirrups. JOGN Nurs 1981;10:104-7.

13 Wright D, Fenwick J, Stephenson P, Monterosso L. Speculum 'self-insertion': a pilot study. Women's Health 2005;14:1098.

14 Williams JG, Park LI, Kline J. Reducing distress associated with pelvic examinations: a stimulus control intervention. Women and Health 1992;18:41-53.

15 Dzuba IG, Diaz EY, Allen B, Leonard YF, Lazcano Ponce EC, Shah KV, et al. The acceptability of self-collected samples for HPV testing vs. the Pap test as alternatives in cervical cancer screening. J Womens Health Gend Based Med 2002;11:265-75.

16 Sellors JW, Lorincz A, Mahony JB, Mielzynska I, Lytwyn A, Roth P, et al. Comparison of self-collected vaginal, vulvar and urine samples with physician-collected cervical samples for human papillomavirus testing to detect high-grade squamous intraepithelial lesions. CMAJ 2000;163:513-8.

17 Institute of Medicine Committee on Quality of Health Care in America. Crossing the quality chasm. Washington, DC: National Academy Press, 2001.

(Accepted 22 May 2006)

doi 10.1136/bmj.38888.588519.55

Department of Family and Community Medicine, Eisenhower Army Medical Center, Fort Gordon, GA 30905, USA

Dean A Seehusen research director

Dawn R Johnson staff physician

J Scott Earwood staff physician

Jamie Cornali family nurse practitioner

Kelly Gillespie staff physician

Maria Doria staff physician

Edwin Farnell IV resident physician

Jason Lanham resident physician

Mathematics and Computer Science Department, Augusta State University, Augusta, GA 30904, USA

Sankar N Sethuraman professor of statistics

Correspondence to: D A Seehusen dseehusen@msn.com 